\title{
notes
}

\section{L'organisation pratique de la radioprotection à Framatome}

\author{
B. BREGEON $\left({ }^{\star}\right)$, M. de KOVALEWSKY $\left({ }^{\star \star}\right)$, J. BINDER $\left({ }^{(\star \star}\right)$ \\ (Manuscrit reçu le 7 juin 1985)
}

\section{PRESENTATION}

Framatome est à la fois une entreprise d'étude et d'ingénierie et un ensemble industriel dont l'objet fondamental est de concevoir, construire et vendre tout ou partie des centrales nucléaires à eau ordinaire, en particulier la chaudière et son combustible.

Framatome doit aussi assurer l'ensemble des services demandés par le client, liés au bon fonctionnement de ces centrales ; son activité s'exerce en France et à l'étranger. La société est découpée en 4 établissements :

- l'établissement de Courbevoie, à la tour Fiat à La Défense, où se trouve en particulier le siège ;

- l'établissement de Lyon dont la vocation est la maintenance des centrales nucléaires ;

- deux établissements de type industriel (Le Creusot et Chalon) dont l'activité est la construction des composants lourds de la chaudière.

Sur le plan de l'organisation de la radioprotection et de la gestion des personnels DATR, les actions du département Radioprotection se développent conformément à la réglementation française en vigueur et se répartissent autour de 5 axes :

- la formation en radioprotection ;

- le suivi médical ;

- le suivi dosimétrique ;

- la gestion des personnels DATR;

- la préparation et l'exécution du travail dans un but de réduction des doses.

\section{LA FORMATION EN RADIOPROTECTION}

Pour la formation des travailleurs, la société s'adresse à des organismes de formation, reconnus pour leur compétence (en sachant qu'il n'existe pas dans ce domaine d'organisme agréé). Ces organismes ont mis au point

(*) Framatome, Service Radioprotection, 10, rue J. Récamier, 69006 Lyon.

$(\star \star)$ Framatome, Gaco-Metra pour Framatome, Service médical, tour Fiat, 92084 Paris-La-Défense Cedex 16.

$\left({ }^{\star \star \star}\right)$ Service inter-entreprise de médecine du travail de Saône-et-Loire, BP 13 , 71380 Saint-Marcel. 
leur module de formation en accord avec le département Sécurité, Radioprotection, Environnement d'EdF. La formation comporte 2 niveaux : agent d'exécution et chef de travaux. Un contrôle des connaissances est effectué à l'issue du stage et un avis est donné à l'employeur, qui en tient compte pour prendre sa décision d'agrément DATR. Mention est faite dans le carnet DATR, édité par le Groupement intersyndical des industries nucléaires (GIIN) et remis à chaque travailleur. En fonction des circonstances, un recyclage est parfois effectué. II est orienté vers les problèmes de radioprotection propres aux interventions de Framatome les plus caractéristiques et adapté au niveau de responsabilité des personnels.

Quant à la formation des médecins, il faut souligner que, bien que dans l'état actuel de la législation française, le certificat d'études spéciales de médecine du travail soit suffisant pour assurer le suivi du personnel DATR, la pratique montre qu'une formation supplémentaire est nécessaire. Cette dernière a donc été assurée :

- par le Service médical du travail du CEA de Fontenay-aux-Roses ;

- ensuite, par le stage organisé à l'intention des médecins du travail par la Société française de radioprotection et le GIIN ; tous les médecins de Framatome ont suivi ce stage ;

- enfin, il existe un CES de Radioprotection appliquée à la médecine du travail à la Faculté de médecine de Paris. Deux médecins de Framatome sont titulaires de ce diplôme.

\section{LE SUIVI MEDICAL}

Compte tenu du fait que les travailleurs sont répartis entre 4 établissements, plus des chantiers, soit de montage, soit de maintenance, répartis sur l'ensemble du territoire national et que Framatome ne possède pas de service médical autonome et doit s'adresser à des services médicaux inter-entreprises (pour les travailleurs d'un établissement, ce sera un service inter-entreprises de sa circonscription, pour les travailleurs des chantiers de montage, ce sera un service inter-entreprises choisi par le comité inter-entreprises d'hygiène et de sécurité du chantier), des dispositions particulières ont dû être prises pour assurer le suivi médical des travailleurs. Ces derniers ont été ainsi répartis en différentes catégories :

- ceux des établissements, qui sont suivis, toujours au même endroit, par le même médecin ;

- ceux des chantiers montage, qui sont suivis par différents services médicaux, dispersés sur l'ensemble du territoire (pour ces salariés, cependant, le temps d'affectation à un chantier précis est suffisamment long - environ 2 ans - et connu à l'avance, ce qui permet de bien organiser la circulation des informations médicales les concernant) ;

- ceux, enfin, qui chargés de la maintenance, tournent entre les différents sites, pour une période d'une durée variable : pour ces derniers, il a été décidé de les faire suivre par l'établissement dont ils dépendent administrativement, ce qui suppose voyages, dépenses et perte de temps au moins 2 fois par an.

La priorité a ainsi été donnée au sérieux du suivi médical sur les contraintes administratives qui en découlent. 
En ce qui concerne les dossiers médicaux, chaque travailleur DATR en a obligatoirement deux :

- le dossier médical spécial qui le suit ;

- le dossier médical classique qui reste la propriété du service médical inter-entreprises (un extrait peut en être adressé à un confrère qui le demande).

Ainsi, aux problèmes de la rotation des travailleurs, s'ajoutent ceux de la rotation des dossiers. Cette difficulté a été levée entre les 5 médecins des 4 établissements qui, d'un commun accord, se communiquent la totalité du dossier. II n'en est pas toujours de même avec les sites.

\section{LE SUIVI DOSIMETRIQUE}

Pour la fourniture des films, Framatome fait appel soit au Service central de protection contre les rayonnements ionisants (SCPRI), soit aux différents laboratoires agréés par lui.

Dans la mesure du possible, il s'agit d'abonnements mensuels. Cependant, pour certaines personnes qui n'interviennent que très rarement, Framatome demande aux laboratoires des films dits banalisés qui ne sont distribués qu'à la demande.

L'utilisation des films règle le problème de la dosimétrie légale, mais ne règle pas pour autant le problème dosimétrique des travailleurs DATR.

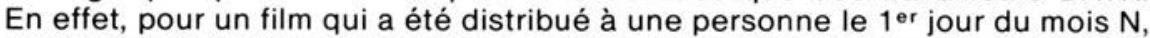
en moyenne on ne connaîtra l'équivalent de dose absorbée par l'intéressé qu'entre le 15 et le 20 du mois $N+2$. L'employeur a donc presque 3 mois de retard pour la connaissance du cumul des doses de son personnel.

II est possible de faire face à ce problème en exploitant la dosimétrie journalière, mais étant donné la diversité des chantiers et la mobilité des gens, le système est difficilement exploitable, sauf au coup par coup. Pour avoir, à l'échelon de la Société, une vision globale de la dosimétrie de son personnel, il a été mis en place un système de dosimétrie par thermoluminescence (pastilles de LiF). Lors de la distribution du film, est placée dans la même pochette une plaquette de 2 pastilles de LiF. Ce système n'est mis en œuvre que pour les personnes qui reçoivent des doses significatives (supérieures à 1500 mrem dans l'année) ou susceptibles de participer à des interventions à haut risque potentiel. II est ainsi possible de dresser un tableau représentant la dosimétrie, cumulée sur 12 mois, avec 10 mois de résultats de films et 2 mois de résultats des pastilles LiF. Ces tableaux transmis à la hiérarchie permettent de prévoir à l'avance, en fonction des doses absorbées, l'utilisation du personnel.

L'employeur est naturellement responsable des dépassements de dose. II lui appartient donc de se donner les moyens de pouvoir faire le bilan à tout moment. Cela ne veut pas dire que l'individu peut se désintéresser des doses qu'il absorbe. Ainsi, chaque travailleur reçoit "un carnet DATR" sur lequel il doit porter des doses journalières ainsi que les doses films qui lui sont communiquées mensuellement. A tout moment, le travailleur peut donc faire le bilan de sa dosimétrie et, si besoin est, alerter sa hiérarchie s'il trouve que le bilan s'alourdit. Compte tenu de tous les 
contrôles qui sont effectués, toute personne qui atteint 2 rem en trois mois ou 4 rem en 12 mois est interdite de zone contrôlée. Son cas est examiné par la hiérarchie et le département Radioprotection. Après un bilan, les entrées en zone contrôlée peuvent être autorisées à nouveau sous réserve d'une autorisation écrite de la hiérarchie comportant la dose maximale à ne pas dépasser dans le mois et des restrictions de travail. Le médecin est destinataire de cette autorisation.

\section{LA GESTION DES PERSONNELS DATR}

En 1984, le département Radioprotection de Framatome gérait environ 1350 DATR appartenant aux 4 établissements de Framatome et aux sociétés Novatome et Fragéma. Cette gestion se fait à l'aide d'un système informatique. Elle s'exerce de façon centralisée de manière à conserver une unité d'action pour l'ensemble des personnels, tout en tenant compte de certaines spécificités propres à chaque établissement dans les domaines suivants :

- abonnement dosimétrique et gestion des abonnements ;

- gestion des doses ;

- tenue à jour du fichier employeur ;

- relance des visites médicales.

\section{LA PREPARATION, L'EXECUTION DU TRAVAIL ET LE RETOUR D'EXPERIENCE}

Les interventions de Framatome dans les centrales nucléaires peuvent être séparées en deux types bien distincts:

- les interventions au titre du montage et de la mise en service industrielle (MSI) ;

- les interventions de maintenance après la MSI.

Pendant la phase de montage et la mise en service industrielle, les problèmes de radioprotection sans être ignorés sont marginaux et les doses sont presque toutes nulles. II n'en est pas de même des interventions dans le cadre de la maintenance. En effet, la majeure partie des interventions s'effectue soit sur le circuit primaire principal, soit sur des circuits en relation directe avec le circuit primaire. L'intervention du département Radioprotection se fait alors à plusieurs niveaux.

Tout d'abord il s'agit de garder en mémoire un certain nombre de données concernant les nuisances radioactives. Ces données sont les débits de dose à certains postes de travail, et les doses intégrées par le personnel pour l'exécution de certaines opérations. Cette banque de données est détenue par le département Radioprotection et les informations proviennent des fiches d'interventions qui sont établies par les techniciens de radioprotection du département au cours de l'assistance qu'ils apportent aux équipes de la maintenance pendant leurs interventions.

Au stade de la préparation des chantiers, pour certains dossiers, une prévision de la dosimétrie est faite, en concertation avec les chefs d'inter- 
vention qui définissent les postes de travail, les gestes et les temps d'intervention. Si nécessaire, une procédure d'intervention sur le plan de la radioprotection est écrite et jointe au dossier. Dans le but d'améliorer les techniques et de diminuer les temps d'intervention pour réduire les doses, des séances d'entraînement sur maquette à l'échelle 1 sont également organisées. Le technicien en radioprotection qui sera présent sur le site assiste aux séances d'entraînement.

Enfin, au niveau de l'exécution pour les interventions importantes, un technicien du département assiste le chef de travaux pour les problèmes de radioprotection concernant le personnel de Framatome et de ses soustraitants. A l'issue de sa mission, il établit un rapport qui fait le bilan dosimétrique de l'opération, et qui fait ressortir les points particuliers qui méritent d'être étudiés en vue de réduire les doses. Par la suite, des réunions ont lieu avec les responsables d'intervention et des décisions sont prises pour améliorer les méthodes ou les matériels.

\section{CONCLUSION}

L'ensemble du système a permis de réduire de manière significative les doses pour certaines opérations répétitives, en donnant lieu parfois à une révision en profondeur des procédés d'intervention. Cependant, globalement, la société doit faire face à une augmentation très rapide de ses interventions, liées à la montée en puissance du parc électronucléaire français (42 réacteurs en service en 1985 contre 8 en 1978).

Pour faire face aux problèmes posés par l'évolution de la dose collective et de la dose individuelle, Framatome a donc engagé des actions portant sur :

- l'étude systématique des postes de travail pénalisants ;

- l'étude des dispositifs à mettre en place pour réduire les doses ;

- la réalisation du matériel ;

- la formation des équipes d'intervention sur le type de matériel ;

- l'entretien du matériel.

Evidemment, l'ensemble de ces actions ne peut être envisagé sans que soit abordé le problème des coûts. Les contraintes posées par la radioprotection et la limitation des doses ne peuvent se traiter à part, mais doivent s'inscrire dans le cadre général de la rentabilité de l'entreprise. D'une manière générale, il apparaît que les grandes actions qui sont menées en vue de la réduction des doses ne peuvent se faire qu'en concertation avec I'utilisateur. II y a eu à Framatome des développements de ce type, il y en aura certainement d'autres, mais tout ne peut pas être pris en considération, ne serait-ce que par le fait que certains problèmes ne peuvent se présenter qu'une fois. 